PROCEEDINGS OF THE

AMERICAN MATHEMATICAL SOCIETY

Volume 131, Number 10, Pages 3053-3057

S 0002-9939(03)06976-4

Article electronically published on April 30, 2003

\title{
UNIFORM PERFECTNESS OF SELF-AFFINE SETS
}

\author{
FENG XIE, YONGCHENG YIN, AND YESHUN SUN
}

(Communicated by Michael Handel)

\begin{abstract}
Let $f_{i}(x)=A_{i} x+b_{i}(1 \leq i \leq n)$ be affine maps of Euclidean space $\mathbb{R}^{N}$ with each $A_{i}$ nonsingular and each $f_{i}$ contractive. We prove that the self-affine set $K$ of $\left\{f_{1}, \ldots, f_{n}\right\}$ is uniformly perfect if it is not a singleton.
\end{abstract}

\section{INTRODUCTION}

For the semi-group $G$ acting on a metric space $(X, d)$, the discontinuous set $\Omega \subset X$ of $G$ consists of points $x$ such that there is an open ball $B$ centered at $x$ such that there are only finitely many $g \in G$ satisfying $g B \cap B \neq \emptyset$. Its complement $\Lambda=X \backslash \Omega$ is called the limit set of $G$.

Let $f_{i}: X \rightarrow X(1 \leq i \leq n)$ be contractive maps of complete metric space $(X, d)$ and let $G=\left\langle f_{1}, \ldots, f_{n}\right\rangle$ be the semigroup generated by $\left\{f_{1}, \ldots, f_{n}\right\}$ and $G_{k}=\left\{f_{j_{1}} \circ \cdots \circ f_{j_{k}} \mid 1 \leq j_{i} \leq n, 1 \leq i \leq k\right\}(k \geq 0)$, where we mean $G_{0}=$ \{Identity map\}. Then there exists a unique nonempty compact set $K$ such that $K=\bigcup_{i=1}^{n} f_{i}(K)$, which is called the attractor of the iterated function system (IFS, for short) $\left\{f_{1}, \ldots, f_{n}\right\}$ and is the limit of $\bigcup_{g \in G_{k}} g X_{0}$ as $k \rightarrow \infty$ for any nonempty compact subset $X_{0} \subset X$ with respect to the Hausdorff metric of $X$. It can be shown that $K$ coincides with the limit set $\Lambda$ of $G$ (Lemma 2.1).

We are concerned with the geometric properties of the attractor $K$. They often exhibit rich structure and give examples of fractals. Such systems have been extensively studied in regards to Hausdorff measure and the dimension of their attractor. If each map in the system is of one-to-one fashion, the attractor is perfect unless it is a singleton (see Theorem[2.11). An example (see Example 1) is given to show that this is not generally true. We expect better geometric properties of the attractor for a more specific family of maps.

Let $f_{i}(x)=A_{i} x+b_{i}(1 \leq i \leq n)$ be affine maps of Euclidean space $\mathbb{R}^{N}$ with each $N \times N$ matrix $A_{i}$ nonsingular and each $f_{i}$ contractive in the sense $\left|f_{i}(x)-f_{i}(y)\right| \leq$ $a_{i}|x-y|$ for all $x, y \in \mathbb{R}^{N}$ and some $0<a_{i}<1$. The attractor for such a system will be called a self-affine set.

Received by the editors February 24, 2002.

2000 Mathematics Subject Classification. Primary 28A78, 28A80.

Key words and phrases. Uniformly perfect set, self-affine set, Hausdorff dimension.

This research was supported by the National Natural Science Foundation of China, Project No. 10171090. 
A compact subset $E$ of Euclidean space $\mathbb{R}^{N}$ is uniformly perfect if $E$ contains at least two points and there exists a constant $c>0$ such that for any point $x_{0} \in E$ and $0<r<\operatorname{diam} E$, the annulus $\left\{x \in \mathbb{R}^{N}|c r \leq| x-x_{0} \mid \leq r\right\}$ meets $E$ (see $[\mathrm{PO}]$ ). For a compact set $E$ of the complex plane $\mathbb{C}, \mathrm{A}$. F. Beardon and Ch. Pommerenke $[\mathrm{BP}]$ relate uniform perfectness to the notion of the hyperbolic metric of $\overline{\mathbb{C}} \backslash E$ being comparable to the reciprocal of the distance to the boundary. There are many other characterizations of uniformly perfect planar sets (see $[\mathrm{Po}]$ ). In the analytical settings, it has been proved that the Julia sets of rational maps, conformal attractors, and Julia sets of rational semigroups with a common Lipschitz constant are uniformly perfect. We refer the reader to [MC], $\mathrm{HM}$, [S2], and [S1] for details.

Our question is whether the self-affine sets are uniformly perfect. The main result of this paper gives an affirmative answer to this question when the attractor is not a singleton. In particular, if each $f_{i}$ is a similitude, then the self-similar set $K$ is either uniformly perfect or a singleton.

Now we come to our main result.

Main Theorem. The self-affine set containing at least two points is uniformly perfect.

Combining this and Corollary 4.2 in [JV], one immediately has the following statement.

Corollary 1.1. The self-affine set containing at least two points has positive Hausdorff dimension.

\section{Perfectness and Properties of AfFine maps}

In this section, we give the proof of the perfectness of attractors of one-toone contractive maps and some necessary preparations for the proof of our Main Theorem.

2.1. Perfectness of attractors. Let $(X, d)$ be a metric space. The $\epsilon$-neighborhood of the subset $A \subset X$, denoted by $N_{\epsilon}(A)$, is defined to be the set $\{x \in X \mid d(x, A)<\epsilon\}$. We need the following lemma to prove the perfectness of attractors of one-to-one contractive maps.

Lemma 2.1. If $\left\{f_{1}, \ldots, f_{n}\right\}$ is a finite family of contractive maps of complete metric space $X$, then $\Lambda=K$.

Proof. For any point $x \in \Omega$, there is an open ball $B$ centered at $x$ such that there are only finitely many $g \in G$ satisfying $g B \cap B \neq \emptyset$.

Denote $X_{0}=\bar{B}, X_{k}=\bigcup_{g \in G_{k}} g X_{0}$. Then $X_{k} \rightarrow K$ in the Hausdorff metric as $k \rightarrow \infty$. We conclude that $X_{k} \cap B=\emptyset$ for large $k$. This implies $x \notin K$.

On the other hand, if $x \notin K$, there is an open ball $B$ centered at $x$ such that $B$ is disjoint with an $\varepsilon_{0}$-neighborhood $N_{\varepsilon_{0}}(K)$ of $K$ for some $\varepsilon_{0}>0$. Denote $X_{0}=\bar{B}, X_{k}=\bigcup_{g \in G_{k}} g X_{0}$. Then $X_{k} \subset N_{\varepsilon_{0}}(K)$ for large $k$. This yields $X_{k} \cap B=\emptyset$ for large $k$ and $\sharp\{g \in G \mid g B \cap B \neq \emptyset\}<\infty$. Hence $x \in \Omega$.

We proved that $\Omega=X \backslash K$, i.e., $\Lambda=K$. 
Theorem 2.1. Let $f_{i}: X \rightarrow X(1 \leq i \leq n)$ be one-to-one contractive maps of complete metric space $(X, d)$ and $K$ be the attractor. Then $K$ is perfect if it is not a singleton.

Proof. Suppose that $K$ has an isolated point $x$. Choose an open ball $B(x, r)$ centered at $x$ with radius $r>0$ such that $B(x, r) \cap K=\{x\}$. From Lemma 2.1, there exists an element $g \in G$ such that $g B\left(x, \frac{1}{2} r\right) \bigcap B\left(x, \frac{1}{2} r\right) \neq \emptyset$. Since $g B\left(x, \frac{1}{2} r\right) \subset$ $B\left(g(x), \frac{1}{2} r\right)$, we have $g(x) \in B(x, r)$ and $g(x)=x$. It follows from $g$ being a one-to-one contractive map that if there is a point in $K$ different from $x$, say $y$, then $g^{k}(y) \rightarrow x$ in $K$ as $k \rightarrow \infty$. This is a contradiction because $x$ is an isolated point.

Theorem 2.1 does not hold if we draw off the one-to-one condition.

Example 1. Let $X=\mathbb{R}^{1}$, and let $f_{1}(x) \equiv 0$ and $f_{2}(x)=\frac{1}{2} x+\frac{1}{2}$. Then $f_{1}$ and $f_{2}$ are contractive, and $K=\left\{0,1, a_{1}, \ldots, a_{n}, \ldots\right\}$, where $a_{n}=\sum_{j=1}^{n} \frac{1}{2^{j}}$.

2.2. Properties of affine maps. Let $A=\left(a_{i j}\right)$ be an $N \times N$ real matrix. The norm of $A$, denoted by $\|A\|$, is defined to be $\sqrt{\sum a_{i j}^{2}}$. Denote by $e^{j}$ the $j^{\text {th }}$ member of the standard basis of $\mathbb{R}^{N}$. It is easy to check that

(1) $\|A B\| \leq\|A\|\|B\|$,

(2) $\|H\|=\sqrt{N}$ for orthogonal matrix $H$, and

(3) $\|A\|=\sqrt{\sum_{j=1}^{N}\left|A\left(e^{j}\right)\right|^{2}}$.

The following lemma plays a crucial role in the proof of our Main Theorem.

Lemma 2.2. Let $f(x)=A x+a$ be a one-to-one linear map of $\mathbb{R}^{N}$. Then there exists $\alpha_{A}>0$ depending only on $A$ such that

$$
\operatorname{diam} \phi \circ f\left(E_{0}\right) \geq \alpha_{A} \operatorname{diam} \phi\left(B_{0}\right),
$$

for any closed ball $B_{0}=\overline{B\left(0, R_{0}\right)}$, subset $E_{0}=\left\{\mathbf{0}, R_{0} e^{1}, \cdots, R_{0} e^{N}\right\}$, and any map $\phi(x)=B x+b$ of $\mathbb{R}^{N}$.

Proof. First note that there is an orthogonal transformation $h(x)=H x$ depending on $\phi$ so that $|\phi \circ h(x)-b|$, or equivalently $|B H x|$, assumes the maximum for $x \in B_{0}$ at $R_{0} e^{1} \in E_{0}$. Therefore,

$$
\begin{aligned}
\operatorname{diam} \phi \circ f\left(E_{0}\right) & =\operatorname{diam} B A\left(E_{0}\right) \\
& \geq \max _{1 \leq j \leq N}\left\{\left|B A\left(R_{0} e^{j}\right)\right|\right\} \geq \frac{R_{0}}{\sqrt{N}}\|B A\| \\
& =\frac{R_{0}\|B A\|\left\|A^{-1} H\right\|}{\sqrt{N}\left\|A^{-1} H\right\|} \geq \frac{R_{0}\|B H\|}{N\left\|A^{-1}\right\|} \\
& \geq \frac{\max _{1 \leq j \leq N}\left\{\left|B H\left(R_{0} e^{j}\right)\right|\right\}}{N\left\|A^{-1}\right\|}=\frac{\left|B H\left(R_{0} e^{1}\right)\right|}{N\left\|A^{-1}\right\|}=\frac{\max _{x \in B_{0}}\{|B H x|\}}{N\left\|A^{-1}\right\|} \\
& =\frac{\operatorname{diam} \phi \circ h\left(B_{0}\right)}{2 N\left\|A^{-1}\right\|}=\frac{1}{2 N\left\|A^{-1}\right\|} \operatorname{diam} \phi\left(B_{0}\right) .
\end{aligned}
$$

Take $\alpha_{A}=\frac{1}{2 N\left\|A^{-1}\right\|}$. 


\section{Proof of the Main Theorem}

Suppose that $K$ is not a singleton. We may assume that the origin lies in $K$ and is the fixed point of some affine map in $\left\{f_{1}, \ldots, f_{n}\right\}$, say, $f_{1}$. Otherwise, we can apply a translation $T(x)=x+\left(A_{1}-I d\right)^{-1} b_{1}$ of $\mathbb{R}^{N}$, where we denote by $I d$ the identity matrix, to conjugate the system to a newer one. This does not change the uniform perfectness. Let $B_{0}$ be the closed ball centered at the origin and of radius $R_{0}$ such that $f_{i}\left(B_{0}\right) \subset B_{0}$ for $1 \leq i \leq n$. Denote by $E_{0}$ the subset $\left\{\mathbf{0}, R_{0} e^{1}, \ldots, R_{0} e^{N}\right\}$.

It follows from Theorem 2.1 that $K$ is perfect and hence contains infinitely many points. If $K$ does not lie in any lower-dimensional subspace of $\mathbb{R}^{N}$, or in other words the nonzero vectors in $K$ span $\mathbb{R}^{N}$, one can find $N$ distinct points $y^{j}(1 \leq j \leq N)$ in $K$ other than the origin, such that the vectors $y^{j}(1 \leq j \leq N)$ are linearly independent. Let $f_{0}(x)=A_{0} x$ be the linear map sending $R_{0} e^{j}$ to $y^{j}$. Hence $A_{0}$ is nonsingular.

On the other hand, if $K \backslash$ zero vector\} spans a lower-dimensional subspace $L$ of $\mathbb{R}^{N}$, then $L$ is invariant under each $f_{i}$ for $1 \leq i \leq n$. Note that a canonical isomorphism between $L$ and some lower-dimensional Euclidean space $\mathbb{R}^{m}(1 \leq m<$ $N)$ conjugates $f_{i}$ 's to affine maps and keeps uniform perfectness. Thus it suffices to consider the former case.

Let $x$ be any point in $K$ and $B(x, r)$ be the closed ball centered at $x$ and of radius $r<\operatorname{diam} K$. There are $g_{k} \in G_{k}$ such that $x \in g_{k+1}\left(B_{0}\right) \subset g_{k}\left(B_{0}\right)$ and $g_{k+1}=g_{k} \circ f_{j_{k+1}}\left(1 \leq j_{k+1} \leq n\right)$ for each $k \geq 0$. Let $k_{0}$ be the smallest integer such that diam $g_{k_{0}}\left(B_{0}\right) \geq r$. Then $g_{k_{0}+1}\left(B_{0}\right) \subset B(x, r)$. Let $\alpha=\min _{0 \leq i \leq n}\left\{\alpha_{A_{i}}\right\}$, where $\alpha_{A_{i}}$ is the constant given for $f_{i}(0 \leq i \leq n)$ in Lemma 2.2 Therefore, $\operatorname{diam} g_{k_{0}+1} \circ f_{0}\left(E_{0}\right) \geq \alpha \operatorname{diam} g_{k_{0}+1}\left(B_{0}\right) \geq \alpha \operatorname{diam} g_{k_{0}+1}\left(E_{0}\right) \geq \alpha^{2} \operatorname{diam} g_{k_{0}}\left(B_{0}\right) \geq$ $\alpha^{2} r$. Let $c=\frac{1}{2} \alpha^{2}$. Some vertex of $g_{k_{0}+1} \circ f_{0}\left(E_{0}\right)$, which lies in $K$, meets the annulus $\left\{y \in \mathbb{R}^{N}|c r \leq| y-x \mid \leq r\right\}$. This completes the proof.

Example 2. One cannot extend the Main Theorem to the one-to-one contractive maps of complete metric space. Let $f_{1}(x)=\frac{1}{3} x^{2}, f_{2}(x)=\frac{1}{3} x^{2}+\frac{2}{3}$, and $X=[0,1]$. Obviously, the attractor of $\left\{f_{1}, f_{2}\right\}$ is a Cantor set. Define $r_{n}$ and $R_{n}$ inductively by $r_{1}=\frac{1}{3}, R_{1}=\frac{2}{3}$ and $r_{n}=\frac{1}{3} r_{n-1}^{2}, R_{n}=\frac{1}{3} R_{n-1}^{2}$ for $n>1$. Then the sequence of annuli (in this case, one interval or two disjoint intervals with equal length) $A_{n}=\left\{x \in X\left|r_{n}<\right| x \mid<R_{n}\right\}$ do not intersect the attractor and all are centered at the origin. Note that $R_{n+1} / r_{n+1}=\left(R_{n} / r_{n}\right)^{2}=\cdots=2^{2^{n}} \rightarrow \infty$ as $n \rightarrow \infty$. This contradicts the definition of uniform perfectness.

\section{ACKNOWLEDGEMENT}

The authors thank the referee for careful reading and helpful comments and suggestions.

\section{REFERENCES}

[BP] A. F. Beardon and Ch. Pommerenke, The Poincaré metric of plane domains, J. London Math. Soc. 18 (1978), 475-483. MR 80a:30020

[CG] L. Carleson and T. Gamelin, Complex Dynamics, Universitext: Tracts in Mathematics, Springer-Verlag, New York, 1993. MR 94h:30033

[Fa] K. Falconer, Fractal geometry: Mathematical foundations and applications, Wiley, New York, 1990. MR 92j:28008 
[HM] A. Hinkkanen and G. J. Martin, Julia sets of rational semigroups, Math. Z. 222 (1996), no. 2, 161-169. MR 98d:30038

[JV] P. Järvi and M. Vuorinen, Uniformly perfect sets and quasiregular mappings, J. London Math. Soc. 54 (1996), no. 2, 515-529. MR 98d:30031

[MC] R. Mañé and L. F. da Rocha, Julia sets are uniformly perfect, Proc. Amer. Math. Soc. 116 (1992), 251-257. MR 92k:58229

[Ma] P. Mattila, Geometry of sets and measures in Euclidean spaces: Fractals and rectifiability, Cambridge University Press, 1995. MR 96h:28006

[Po] Ch. Pommerenke, Uniformly perfect sets and the Poincaré metric, Arch. Math 32 (1979), 192-199. MR 80j:30073

[S1] R. Stankewitz, Uniformly perfect sets, rational semigroups, Kleinian groups and IFS's, Proc. Amer. Math. Soc. 128 (2000), no. 9, 2569-2575. MR 2000m:37075

[S2] Uniformly perfect analytic and conformal attractor sets, Bull. London Math. Soc. 33 (2001), no. 3, 320-330. MR 2001m:37095]

Department of Mathematics, Zhejiang University, Hangzhou, Zhejiang 310027, PeoPLE'S REPUBlic OF CHINA

E-mail address: xiefengmath@hotmail.com

Current address: 420 Temple St., \#517, New Haven, Connecticut 06511

E-mail address: feng.xie@yale.edu

Department of Mathematics, Zhejiang University, Hangzhou, Zhejiang 310027, PeoPle's Republic of China - And - Morningside Center of Mathematics, Chinese Academy of Sciences, Beijing 100080, People's Republic of China

E-mail address: yin@math.zju.edu.cn

Department of Mathematics, Zhejiang University, Hangzhou, Zhejiang 310027, PeoPLE'S REPUBLic OF CHINA

E-mail address: sun@math.zju.edu.cn 